\title{
Aumento nas reclamações de idosos sobre a saúde suplementar no Brasil
}

\author{
Increase in the complaints of the elderly about supplemental health \\ in Brazil
}

Natália Carolina de Sousa (https://orcid.org/0000-0002-1393-3489) ${ }^{1}$

Rosana Rosseto de Oliveira (https://orcid.org/0000-0003-3373-1654) ${ }^{1}$

Maria Aparecida Salci (https://orcid.org/0000-0002-6386-1962) ${ }^{1}$

Ligia Carreira (https://orcid.org/0000-0003-3891-4222) ${ }^{1}$

Thamires Fernandes Cardoso da Silva Rodrigues (https://orcid.org/0000-0001-7942-4989) ${ }^{1}$

Cremilde Aparecida Trindade Radovanovic (https://orcid.org/0000-0001-9825-3062) ${ }^{1}$

\footnotetext{
${ }^{1}$ Universidade Estadual de

Abstract The scope of this study was to describe the complaints of elderly Brazilians received by the National Supplemental Health Agency. It is an ecological study, with secondary data from the Integrated Inspection System. A trend analysis of the complaints received by the National Supplemental Health Agency of elderly beneficiaries from all over Brazil was conducted between 2008 and 2017. A total of 111,497 complaints were filed by elderly beneficiaries of the supplemental health system. The highest number of complaints (27.07) and the highest average annual increase (7.79, $\left.r^{2}=0,97\right)$, and the South region, with the lowest average rate for the period (7.11) and the lowest observed mean annual increase $\left(1.84 ; r^{2}=0.92\right)$ were identified. The highest rate of complaints was related to the coverage of the plan, between the elderly aged 70 to 79 and beneficiaries of individual or family plans. Growing dissatisfaction among older people with the supplemental health system reflected the large number of complaints received by the National Supplemental Health Agency over a period of nine years.

Key words Supplemental Health, Elderly, Comprehensive Healthcare, Epidemiologic Methods
Resumo O objetivo foi descrever as reclamações de idosos brasileiros recebidas pela Agência Nacional de Saúde Suplementar. Trata-se de um estudo ecológico, com dados secundários provenientes do Sistema Integrado de Fiscalização. Realizou-se análise de tendência das reclamações recebidas pela Agência Nacional de Saúde Suplementar de beneficiários idosos de todo o Brasil, no periodo de 2008 a 2017. Analisaram-se 111.497 reclamações realizadas por idosos beneficiários da saúde suplementar. Identificou-se aumento das taxas de reclamações para todas as regiões do país, com destaque para a região Nordeste, que apresentou a maior taxa média de reclamações $(27,07)$ e o maior aumento médio anual $\left(7,79 ; r^{2}=0,97\right)$, e a região Sul, com a menor taxa média para o periodo $(7,11)$ e o menor aumento médio anual observado $\left(1,84 ; r^{2}=0,92\right)$. A maior taxa de reclamações foi relacionada à cobertura do plano, entre idosos de 70 a 79 anos e beneficiários de planos individuais ou familiares. A crescente insatisfação de idosos com o sistema de saúde suplementar refletiu no grande número de reclamações recebidas pela Agência Nacional de Saúde Suplementar ao longo de nove anos.

Palavras-chave Saúde Suplementar, Idoso, Assistência Integral à Saúde, Métodos Epidemiológicos 


\section{Introdução}

No Brasil, a saúde suplementar é definida como todo o atendimento de saúde privado. Este coexiste com os serviços públicos já ofertados pelo Estado há mais de 70 anos, podendo ser assim considerado um "sistema de saúde duplicado", o que também ocorre em outros países como Espanha, Itália, Grécia, Portugal e Reino Unidoํ. Seu marco regulatório ocorreu com o estabelecimento da Lei no 9.656 , de 3 de junho de $1998^{2}$, a qual dispõe sobre os planos e seguros privados de assistência à saúde e a Lei no 9.961, de 28 de janeiro de $2000^{3}$, que criou a Agência Nacional de Saúde Suplementar (ANS), vinculada ao Ministério da Saúde (MS), com a finalidade de uniformizar regras, defender o interesse público na assistência suplementar e regular as operadoras de saúde.

Nos últimos anos, a saúde suplementar passou por uma redução no número de operadoras vigentes e de novos beneficiários. Dentre os principais fatores, destaca-se o fato de que o país vem enfrentando um quadro de recessão econômica, cuja desaceleração da criação de empregos formais, incide negativamente sob as contratações de planos coletivos e individuais ${ }^{4}$. Dados fornecidos pela ANS demonstram que no ano de 2016, houve uma queda de crescimento de $3,2 \%$ dos beneficiários em geral ${ }^{5}$. Entretanto, apesar desta diminuição, as operadoras movimentaram cerca de R $\$ 139,5$ bilhões no ano de 2015, montante este, superior ao orçamento disponibilizado pelo MS no referido ano (R \$ 109,2 bilhões), evidenciando a importância deste mercado no país ${ }^{6}$. Vale mencionar que este valor não se aplica aos planos hospitalares com obstetrícia.

Contrariamente ao encolhimento do setor, o número de beneficiários idosos mostra-se crescente. Tal fato associa-se ao envelhecimento da população brasileira ${ }^{7}$. Estimativas realizadas pelo Instituto Brasileiro de Geografia e Estatística (IBGE), apontam que no ano de 2017, o país possuía aproximadamente 26 milhões de pessoas com mais de 60 anos e em 2050, este número chegará a 66 milhões 8 . O incremento no número de idosos incide sobre os serviços de saúde públicos e privados, visto que estas pessoas possuem necessidades específicas e, frequentemente, múltiplas doenças crônicas, as quais demandam por mais recursos e, consequentemente, mais gastos ao setor de saúde ${ }^{9,10}$.

Nessa perspectiva, o idoso revela-se um cliente oneroso para as operadoras, e para minimizar os encargos oriundos, alguns planos de saúde imputam barreiras que dificultam o seu ingresso e o uso da rede assistencial, além de altos reajustes de mensalidade, gerando um ponto de tensão entre os clientes e as operadoras. Considerando estes elementos é de se esperar que a insatisfação dos idosos culmine em reclamações, as quais são encaminhadas à $\mathrm{ANS}^{10}$. Nesse sentido, vale mencionar que reclamação consiste na "manifestação de desagrado sobre um serviço prestado pela operadora de planos privados de assistência à saúde"10.

Frente ao exposto, a pertinência dessa discussão para a política de saúde no Brasil está relacionada ao contexto de envelhecimento populacional e ao aumento do número de beneficiários idosos ${ }^{10}$. Além disso, a investigação do conceito de qualidade na saúde suplementar sob a perspectiva dos diferentes grupos torna-se de fundamental importância no desenvolvimento e aprimoramento das análises e divulgação de informações do setor ${ }^{11}$. Desta forma, o estudo teve como objetivo descrever as reclamações de idosos brasileiros recebidas pela Agência Nacional de Saúde Suplementar.

\section{Método}

Trata-se de um estudo descritivo, do tipo ecológico realizado a partir dos registros das reclamações de beneficiários idosos do sistema de saúde privado do Brasil, no período de 2008 a 2017. Os dados utilizados são de domínio público, obtidos no Sistema Integrado de Fiscalização (SIF), o qual é gerido pela ANS e hospeda todas as reclamações direcionadas ao setor. $\mathrm{O}$ referido banco de dados possui os registros dos contatos efetuados por beneficiários para a ANS, os quais buscam receber informações para suas dúvidas ou realizar reclamações.

Optou-se por iniciar a análise em 2008, apesar do banco de dados existir desde 2007, devido ao insignificante percentual de informações existentes no primeiro ano de disponibilização dos dados. Foram classificados como idosos as pessoas com idade igual ou superior a 60 anos, conforme disposto no artigo primeiro do Estatuto do Idoso, Lei 10.741/2003 ${ }^{12}$.

Para a seleção dos dados, inicialmente foram filtradas somente as reclamações realizadas, sendo excluídos os contatos referentes à busca de informações ou sem preenchimento. Dentre estes, foram retirados os beneficiários cujos registros não constavam o local de residência, com idade inferior aos 60 anos ou sem preenchimento, dados duplicados e os referentes ao ano de 2007 (Figura 1). 


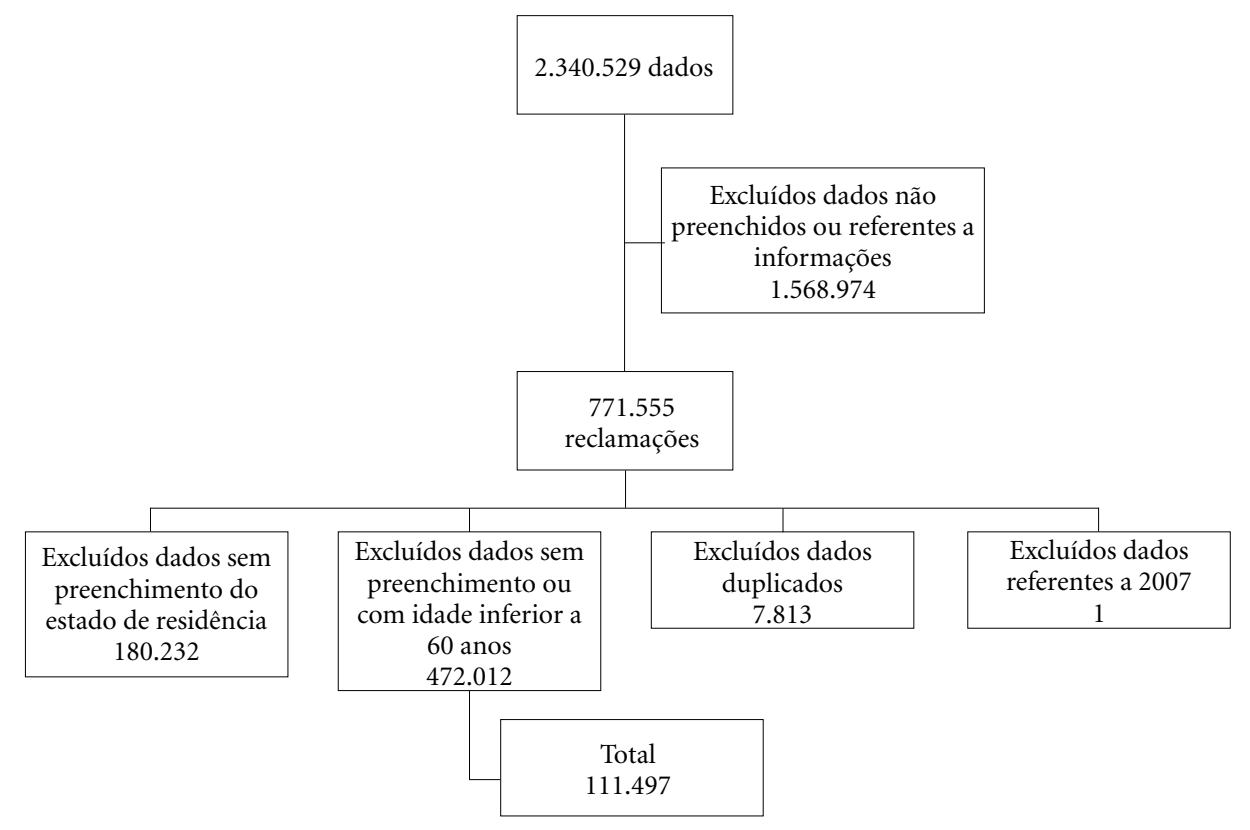

Figura 1. Processo de identificação das reclamações de beneficiários da ANS.

Fonte: Elaborado pelas autoras.

O sistema possui inúmeras variáveis que podem ser acessadas, entretanto optou-se por utilizar as que responderiam ao objetivo deste estudo, sendo: contratação do plano (classificado conforme o vínculo do beneficiário com a operadora em individual ou coletivo, por adesão ou coletivo empresarial), local de residência (unidade de federação de residência do beneficiário), ano de referência (data na qual ocorreu a reclamação), data de nascimento (utilizada para a realização do cálculo da idade do beneficiário) e tema da reclamação (referente à relação do beneficiário com a operadora, classificado em: cobertura; contratos e regulamentos; mensalidades e reajustes, e outros assuntos). Destaca-se que no momento da coleta, o sistema não disponibilizou a variável escolaridade, de modo que não foi utilizada neste estudo.

Para o cálculo da taxa de reclamações, o número de beneficiários foi retirado do tabulador oficial da ANS (Tabnet), sendo sempre escolhidos os dados correspondentes ao mês de dezembro de cada ano analisado. Para a quantificação das taxas de reclamações por beneficiários, utilizouse a razão entre o número de reclamações pelo número de beneficiários de cada ano, multiplicados por 10.000 .

A apresentação dos dados segundo idade e região de residência do beneficiário foi realizada em dois triênios (2008-2010 e 2011-2013) e um quadriênio (2014-2017). Adotou-se tal divisão, pois no início do período analisado a implantação do sistema de notificação das reclamações era recente e pouco consolidada, já no último quadriênio, notou-se maior homogeneidade das notificações. Bem como, por se analisar um período ímpar, cuja simetria não seria possível.

Para a análise de tendência foi utilizada a modelagem de regressão polinomial, considerando as taxas de reclamações como variáveis dependentes (y) e os anos de estudo como variável independente $(\mathrm{x})$. A variável "ano" foi transformada na variável ano centralizada $(x-2012)$ e as séries foram suavizadas por meio de média móvel de três pontos. As análises foram realizadas por meio do programa estatístico SPSS v.21.0.

Por tratar-se de uma pesquisa com dados secundários disponíveis em plataforma pública, foi solicitada a dispensa do termo de consentimento livre e esclarecido junto ao Comitê Permanente 
de Ética em Pesquisa Envolvendo Seres Humanos da Universidade Estadual de Maringá, obtendo aprovação.

\section{Resultados}

Analisaram-se 111.497 queixas realizadas por idosos beneficiários da saúde suplementar no Brasil, no período de 2008 a 2017, correspondendo a uma taxa representativa de 20,12 reclamações por 10 mil beneficiários.

$\mathrm{Na}$ análise de regressão polinomial, identificou-se tendência de aumento nas taxas de reclamação durante o período analisado, em todo o país, passando de 0,24 em 2008 para 30,70 reclamações por 10 mil beneficiários em 2017, com destaque para 2015 que atingiu o ápice de 37,47 reclamações por 10 mil beneficiários. Dentre as regiões do país, o Nordeste sobressaiu-se das demais, com uma taxa de reclamação de $60,33 \mathrm{em}$ 2015 e 53,01 por 10 mil beneficiários em 2016, apresentando a maior taxa média de reclamações (27,07 reclamações por 10 mil beneficiários) e o maior aumento médio anual $\left(7,79 ; \mathrm{r}^{2}=0,97\right)$. Já a região Sul exibiu a menor taxa média para o período (7,11 reclamações por 10 mil beneficiários) e o menor aumento médio anual $\left(1,84 ; \mathrm{r}^{2}=0,92\right)$ (Figura 2).

Verificou-se que o quantitativo de beneficiários idosos se expandiu no país ao longo dos anos, passando de 14.591 .436 entre 2008 e 2010, para 24.477.429 entre 2014 e 2017, exibindo um crescimento anual de $67,75 \%$. Constatou-se também, a predominância de reclamações na faixa etária de 70 a 79 anos, com taxa de reclamação de 34,03 por 10 mil beneficiários. Entretanto, é possível observar aumento do percentual de reclamação entre os idosos com 80 anos e mais, a qual passou de 0,91 no primeiro triênio estudado, para 32,45 no último quadriênio, o que corresponde a um aumento de $25,65 \%$ (Tabela 1 ).

Quanto às modalidades de planos, o individual ou familiar sobressaiu-se totalizando a metade das reclamações (Tabela 2).

$\mathrm{Na}$ análise descritiva sobre os tipos de queixas realizadas por idosos, observou-se a predominância dos eixos de cobertura $(78,95 \%)$, contratos e regulamentos $(12,16 \%)$, seguidos por mensalidades e reajustes $(6,86 \%)$. Esta realidade não se diferencia entre as regiões do país (Tabela 3).

\section{Discussão}

A partir dos resultados deste estudo, pode-se identificar que a taxa de reclamação de idosos brasileiros recebidas pela Agência Nacional de Saúde Suplementar é crescente em todo o país, com destaque para as regiões Nordeste e Sul, sendo a primeira com a maior taxa média de reclamação do Brasil. Nota-se que, apesar do número de beneficiários em geral ter diminuído, houve aumento entre os beneficiários com idade igual ou superior a 60 anos. E, embora a maior taxa de reclamação seja na faixa etária entre 70 e 79 anos, detectou-se crescimento de $25 \%$ das reclamações entre os idosos com 80 anos ou mais. Quanto ao motivo das reclamações, detectou-se que estas se relacionaram principalmente às contratações de planos individuais ou familiares e eram referentes à cobertura.

Estes dados assemelham-se ao encontrado em um estudo conduzido na região Sudeste do país, cujos autores identificaram que no período de 2010 a 2012, a ANS recebeu 92.235 reclamações, das quais $24,2 \%$ foram realizadas por idosos. Além disso, esta população de idosos reclamou duas vezes mais do que as pessoas entre $20 \mathrm{e}$ 59 anos $(60,80$ reclamações por 10 mil beneficiários contra $31,1 / 10 \mathrm{mil})^{10}$.

O aumento na taxa de reclamação de idosos sobre a saúde suplementar, reflete a constante tensão entre a oferta de planos pelas operadoras e o interesse dos beneficiários, cuja lógica de acúmulo de capital consiste no principal determinante das ações e investimentos do setor ${ }^{13}$. Tal lógica desconsidera as especificidades dos sujeitos, fragmentando o cuidado devido às altas especialidades, ignorando as demandas oriundas da senilidade e os determinantes sociais que incidem sobre o processo saúde-doença, o que pode gerar descontentamento nos contratantes 9 .

Revisão sistemática conduzida para avaliar a qualidade dos serviços públicos e privados de saúde em países de média e baixa renda constatou que, apesar de o setor privado possuir melhor desempenho comparativo nos quesitos disponibilidade, capacidade de resposta e esforço, estes são mais propensos a fornecer serviços de baixa qualidade. A qualidade é ainda pior frente às doenças crônicas não transmissíveis, visto que, exigem articulação entre os diferentes níveis de atenção, requerem qualidade estrutural, disponibilidade e competência dos provedores ${ }^{14}$.

Sabe-se que a população idosa possui maior prevalência de doenças crônicas como hipertensão arterial, diabetes mellitus, insuficiência 


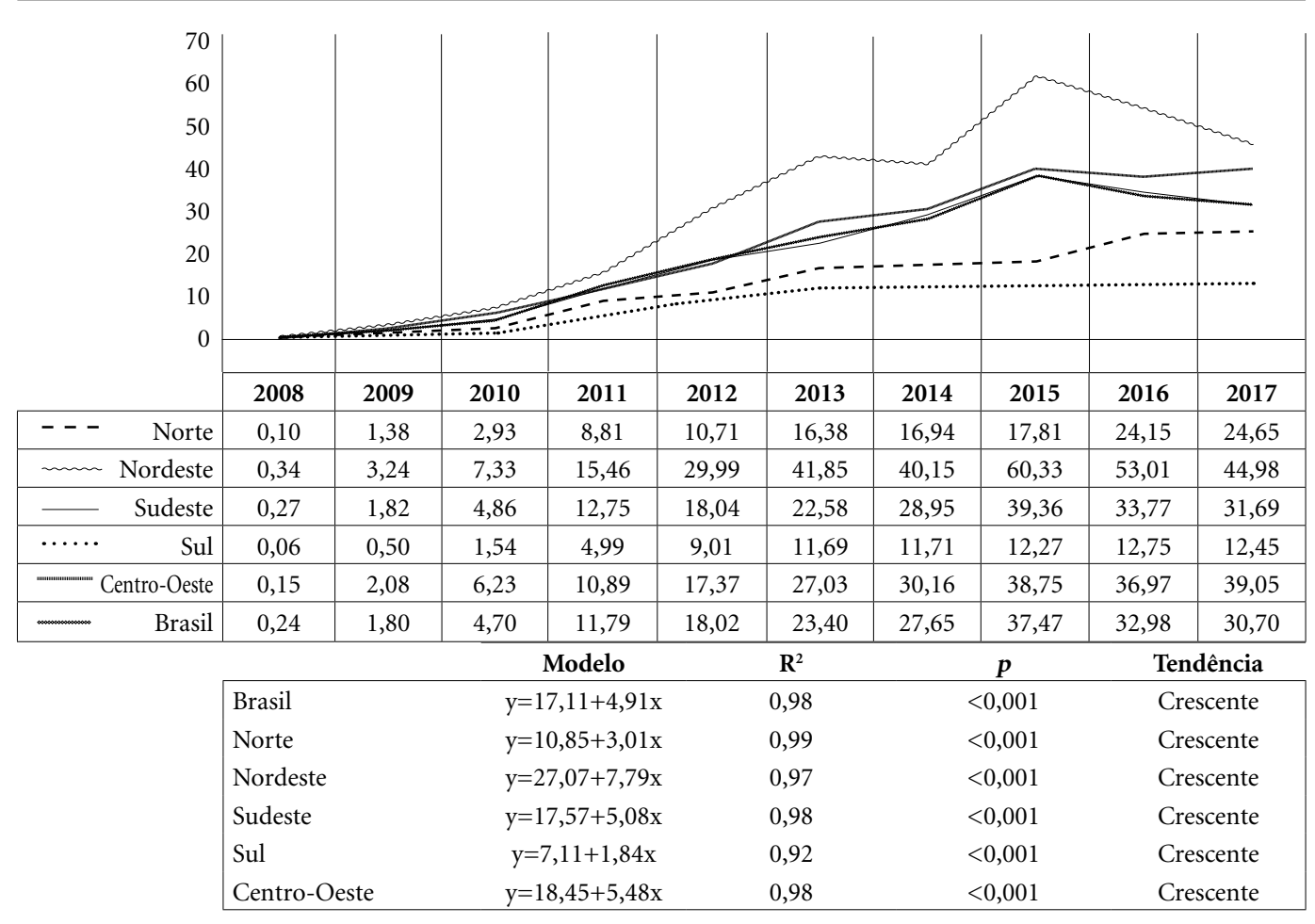

Figura 2. Tendência das taxas de reclamações de idosos por região. Brasil, 2008 a 2017.

Fonte: Elaborado pelas autoras.

Tabela 1. Beneficiários e reclamações por região e período. Brasil, 2008 a 2017.

\begin{tabular}{|c|c|c|c|c|c|c|c|c|c|c|c|c|}
\hline \multirow{2}{*}{$\begin{array}{l}\text { Beneficiário e } \\
\text { Reclamações }\end{array}$} & \multicolumn{4}{|c|}{ 2008-2010 } & \multicolumn{4}{|c|}{ 2011-2013 } & \multicolumn{4}{|c|}{ 2014-2017 } \\
\hline & $\mathrm{Nb}$ & $\mathrm{Nr}$ & $\%$ & $\mathbf{T x}$ & $\mathrm{Nb}$ & $\mathrm{Nr}$ & $\%$ & $\mathbf{T x}$ & $\mathrm{Nb}$ & $\mathrm{Nr}$ & $\%$ & $\mathbf{T x}$ \\
\hline \multicolumn{13}{|l|}{ 60-69 anos } \\
\hline Brasil & 4633002 & 1818 & 53,71 & 3,92 & 8675585 & 15340 & 52,43 & 17,68 & 13093919 & 40784 & 51,72 & 31,15 \\
\hline Norte & 101394 & 26 & 1,43 & 2,56 & 222523 & 219 & 1,43 & 9,84 & 345960 & 691 & 1,69 & 19,97 \\
\hline Nordeste & 534165 & 347 & 19,09 & 6,50 & 1021890 & 3123 & 20,36 & 30,56 & 1574440 & 7581 & 18,59 & 48,15 \\
\hline Centro-Oeste & 195483 & 110 & 6,05 & 5,63 & 430022 & 805 & 5,25 & 18,72 & 711436 & 2413 & 5,92 & 33,92 \\
\hline Sul & 622483 & 89 & 4,90 & 1,43 & 1258696 & 1069 & 6,97 & 8,49 & 1947743 & 2379 & 5,83 & 12,21 \\
\hline Sudeste & 3179477 & 1246 & 68,54 & 3,92 & 5742454 & 10124 & 66,00 & 17,63 & 8514340 & 27720 & 67,97 & 32,56 \\
\hline \multicolumn{13}{|l|}{ 70-79 anos } \\
\hline Brasil & 4845623 & 1100 & 32,50 & 2,27 & 4934285 & 9045 & 30,91 & 18,33 & 7170903 & 24400 & 30,94 & 34,03 \\
\hline Norte & 108811 & 18 & 1,64 & 1,65 & 112926 & 164 & 1,81 & 14,52 & 162610 & 391 & 1,60 & 24,05 \\
\hline Nordeste & 555705 & 198 & 18,00 & 3,56 & 562559 & 1636 & 18,09 & 29,08 & 845285 & 4354 & 17,84 & 51,51 \\
\hline Centro-Oeste & 211250 & 55 & 5,00 & 2,60 & 227433 & 472 & 5,22 & 20,75 & 349717 & 1472 & 6,03 & 42,09 \\
\hline Sul & 675491 & 50 & 4,55 & 0,74 & 667672 & 588 & 6,50 & 8,81 & 1006709 & 1361 & 5,58 & 13,52 \\
\hline Sudeste & 3294366 & 779 & 70,82 & 2,36 & 3363695 & 6185 & 68,38 & 18,39 & 4806582 & 16822 & 68,94 & 35,00 \\
\hline \multicolumn{13}{|l|}{80 e mais } \\
\hline Brasil & 5112811 & 467 & 13,80 & 0,91 & 2743793 & 4873 & 16,66 & 17,76 & 4212607 & 13670 & 17,34 & 32,45 \\
\hline Norte & 119338 & 7 & 1,50 & 0,59 & 58811 & 93 & 1,91 & 15,81 & 88899 & 172 & 1,26 & 19,35 \\
\hline Nordeste & 594740 & 89 & 19,06 & 1,50 & 327822 & 891 & 18,28 & 27,18 & 512298 & 2613 & 19,11 & 51,01 \\
\hline Centro-Oeste & 229367 & 25 & 5,35 & 1,09 & 112229 & 173 & 3,55 & 15,41 & 175422 & 607 & 4,44 & 34,60 \\
\hline Sul & 722027 & 10 & 2,14 & 0,14 & 338490 & 298 & 6,12 & 8,80 & 534632 & 554 & 4,05 & 10,36 \\
\hline Sudeste & 3447339 & 336 & 71,95 & 0,97 & 1906441 & 3418 & 70,14 & 17,93 & 2901356 & 9724 & 71,13 & 33,52 \\
\hline
\end{tabular}

$\mathrm{Nb}$ - Número de beneficiários; $\mathrm{Nr}$ - Número de reclamações; Tx - Taxas de reclamações.

Fonte: Elaborado pelas autoras. 
Tabela 2. Reclamações por modalidade de plano e região. Brasil, 2008 a 2017.

\begin{tabular}{|c|c|c|c|c|c|c|c|c|c|c|c|c|}
\hline \multirow{2}{*}{ Modalidade de plano } & \multicolumn{2}{|c|}{ Norte } & \multicolumn{2}{|c|}{ Nordeste } & \multicolumn{2}{|c|}{ Centro-Oeste } & \multicolumn{2}{|c|}{ Sul } & \multicolumn{2}{|c|}{ Sudeste } & \multicolumn{2}{|c|}{ Brasil } \\
\hline & $\mathbf{n}$ & $\%$ & $\mathbf{n}$ & $\%$ & $\mathbf{n}$ & $\%$ & $\mathbf{n}$ & $\%$ & $\mathrm{n}$ & $\%$ & $\mathbf{n}$ & $\%$ \\
\hline Coletivo Empresarial & 629 & 35,32 & 5195 & 24,94 & 2267 & 36,97 & 1846 & 28,85 & 19730 & 25,84 & 29667 & 26,61 \\
\hline Coletivo por Adesão & 222 & 12,46 & 2420 & 11,62 & 1822 & 29,71 & 960 & 15,00 & 13261 & 17,37 & 18685 & 16,76 \\
\hline Individual ou Familiar & 859 & 48,23 & 11999 & 57,60 & 1832 & 29,88 & 3361 & 52,53 & 40283 & 52,76 & 58334 & 52,32 \\
\hline Dupla cobertura & 1 & 0,06 & 12 & 0,06 & 3 & 0,05 & - & - & 10 & 0,01 & 26 & 0,02 \\
\hline Não identificado & 70 & 3,93 & 1206 & 5,79 & 208 & 3,39 & 231 & 3,61 & 3070 & 4,02 & 4785 & 4,29 \\
\hline Total & 1781 & 100,00 & 20832 & 100,00 & 6132 & 100,00 & 6398 & 100,00 & 76354 & 100,00 & 111497 & 100,00 \\
\hline
\end{tabular}

Fonte: Elaborado pelas autoras.

Tabela 3. Reclamações por tipo de queixa e região. Brasil, 2008 a 2017.

\begin{tabular}{|c|c|c|c|c|c|c|c|c|c|c|c|c|}
\hline \multirow{2}{*}{ Tipo de queixa } & \multicolumn{2}{|c|}{ Brasil } & \multicolumn{2}{|c|}{ Norte } & \multicolumn{2}{|c|}{ Nordeste } & \multicolumn{2}{|c|}{ Centro-Oeste } & \multicolumn{2}{|c|}{ Sul } & \multicolumn{2}{|c|}{ Sudeste } \\
\hline & $\mathbf{n}$ & $\%$ & $\mathbf{n}$ & $\%$ & n & $\%$ & $\mathbf{n}$ & $\%$ & $\mathbf{n}$ & $\%$ & n & $\%$ \\
\hline Cobertura & 88621 & 79,48 & 1417 & 79,56 & 16822 & 80,75 & 4864 & 79,32 & 5236 & 81,84 & 60282 & 78,95 \\
\hline Contratos e Regulamentos & 14177 & 12,72 & 208 & 11,68 & 2283 & 10,96 & 937 & 15,28 & 703 & 10,99 & 10046 & 13,16 \\
\hline Mensalidades e Reajustes & 7626 & 6,84 & 147 & 8,25 & 1542 & 7,40 & 299 & 4,88 & 397 & 6,21 & 5241 & 6,86 \\
\hline Outros tipos de queixas & 1031 & 0,92 & 8 & 0,45 & 176 & 0,84 & 30 & 0,49 & 57 & 0,89 & 760 & 1,00 \\
\hline Não informado & 42 & 0,04 & 1 & 0,06 & 9 & 0,04 & 2 & 0,03 & 5 & 0,08 & 25 & 0,03 \\
\hline Total & 111497 & 100,00 & 1781 & 100,00 & 20832 & 100,00 & 6132 & 100,00 & 6398 & 100,00 & 76354 & 100,00 \\
\hline
\end{tabular}

Fonte: Elaborado pelas autoras.

cardíaca, entre outras ${ }^{15}$. Estudo realizado no Brasil, entre 1998 e 2013, identificou que a taxa de morbidade por doenças do aparelho circulatório entre as pessoas com idade superior a 60 anos foi de $2.995,9$ para cada 100 mil idosos, as neoplasias alcançaram a taxa de $1.195,4$ para cada 100 mil idosos e as doenças do aparelho circulatório $1.876,2$ para cada 100 mil idosos ${ }^{16}$. Nesse contexto, a inabilidade do setor privado em gerir tais patologias, que demandam por ações de médio a longo prazo, inclusive ações de promoção a saúde, podem ter impactado no número de reclamações recebidas pela ANS.

Nesta concepção, pesquisa conduzida em Minas Gerais para analisar o interesse dos envolvidos na oferta, utilização e regulação de programas de promoção da saúde no setor suplementar, detectou que estes programas estão atrelados à lógica de acumulação de capital e lucratividade. Isso porque, a concepção mercadológica sobressai-se sobre o interesse de qualificar a assistência e promover a qualidade de vida dos beneficiá$\operatorname{rios}^{13}$.

Quanto às variações regionais nas taxas de reclamação, tal resultado pode relacionar-se às diferenças locais de renda, escolaridade, saneamento básico e condições de saúde. De modo que a região Sul e Sudeste detém os melhores indicadores socioeconômicos e de saúde, maior densidade tecnológica e oferta de serviços ${ }^{17}$. Inquérito domiciliar realizado no Brasil, no ano de 2013, identificou que o nível de escolaridade e o vínculo empregatício relacionam-se a maior contratação de planos de saúde ${ }^{18}$, bem como a compra de planos de melhor qualidade, que oferecem mais benefícios aos usuários ${ }^{1}$. Estes fatores corroboram os achados deste estudo, cuja região Sul demonstrou a menor taxa de reclamação.

Contrariamente, a região Nordeste sofre desfavoravelmente com tais indicadores, porquanto as taxas de reclamações podem associar-se às famílias com menor poder aquisitivo que adquiriram planos de saúde básicos que possuem cobertura limitada, os quais não oferecem determinados exames ou procedimentos ${ }^{19}$, gerando descontentamento. A expansão de planos de saúde populares oferece experiências negativas para a clientela quanto ao tempo de espera, garantia de atendimento e qualidade do serviço, além de acarretar o aumento das despesas diretas para sua utilização ${ }^{20}$.

Idosos e pacientes com problemas crônicos ou graves são os mais atingidos por restrições impostas pelo mercado ${ }^{21}$. Estudo de revisão teórica 
detectou que no ano de 2013, 3\% dos beneficiários contrataram planos com a menor pontuação recebida pelo Índice de Desempenho da Saúde Suplementar (IDSS), refletindo sua baixa qualidade ${ }^{1}$.

Outro aspecto que possivelmente contribuiu para a diferença nas taxas de reclamações nas regiões citadas trata-se do nível de dependência entre os idosos dessas localidades, porquanto quanto maior o grau de dependência mais se demanda por recursos de saúde, os quais podem não satisfazer integralmente os anseios desta população. Estudo identificou que as pessoas com idade superior a 60 anos residentes no Nordeste do país, possuíam maior nível de dependência funcional, quando comparados com os demais. Os autores observaram que as maiores prevalências se associavam às áreas com condições socioeconômicas menos satisfatórias ${ }^{22}$.

No tocante ao aumento dos beneficiários com idade superior a 60 anos detectados neste estudo, este associa-se, em parte, ao envelhecimento populacional. Os idosos representam cerca de $10,8 \%$ da população e, a expectativa de vida, nessa faixa etária, é de aproximadamente 22,7 anos ${ }^{23}$. Prevê-se que, em 2040, haverá uma inversão da pirâmide etária, cuja esperança de vida ao nascer será de 79,9 anos, com a proporção de 153 idosos para cada 100 pessoas menores de 15 anos, o número de pessoas com mais de 60 anos, passará de 1.234.176 em 1920 para 54.204.894 em 2040 ${ }^{16}$. Estes números implicam em um crescimento do índice de envelhecimento com concomitante redução da razão de dependência, resultando em consideráveis encargos assistenciais para todos os setores da sociedade ${ }^{16}$.

Observou-se também que, os idosos com idade entre 70 e 79 anos, detinham a maior taxa de reclamação sobre o setor de saúde privado, concomitante, houve crescimento significativo de reclamações entre os idosos acima de 80 anos. De forma semelhante, estudo verificou que com o avançar da idade, o coeficiente de reclamação é maior, variando entre 57,9 reclamações/10.000 beneficiários de 65-69 anos até 63,9 reclamações/10.000 beneficiários de 74-79 anos ${ }^{12}$.

Sabe-se que o crescimento exponencial de idosos, representa um desafio para o país. A saúde suplementar será afetada, porquanto o aumento no número de idosos e na sua expectativa de vida, elevam as chances de adquirirem doenças, incapacidades e/ou sequelas, as quais exigem maiores custos para as operadoras. Estudo conduzido em Santa Catarina, com 599 participantes com idade superior a 60 anos, identificou que as mulheres possuíam a média de 3,8 doenças crônicas e os homens 2,8; as patologias mais prevalentes foram a Hipertensão Arterial Sistêmica, Doenças Coronarianas, Artrite/Reumatismo/Artrose e Depressão. Dentre os entrevistados, 380 apresentaram algum nível de incapacidade funcional ${ }^{24}$. Para atender às necessidades da população, faz-se necessário planejar as políticas a médio e longo prazo, que possam permitir transformações nos setores econômicos, de saúde, social e mercado de trabalho ${ }^{16}$.

Quanto ao tipo de contrato, os planos individuais e familiares apresentaram a maior porcentagem de reclamações realizadas por idosos. Alguns aspectos regulatórios incidem sobre esta modalidade, tornando-a mais frágil no que se refere ao equilíbrio do poder de negociação dos contratantes $^{25}$, o que pode ter contribuído com os achados deste estudo.

As principais queixas foram referentes às temáticas de cobertura e contratos. Os resultados corroboram estudos conduzidos no Brasil com beneficiários idosos ${ }^{10}$ e na população em ge$\mathrm{ral}^{11,21}$. As operadoras limitam a cobertura e modificam a execução contratual já consolidadas, para que novas interpretações desfavoráveis ao consumidor sejam consideradas ${ }^{26}$. Assim, cada vez mais impõem-se restrições de coberturas e exigências rigorosas para autorização de serviços aos contratantes ${ }^{26}$.

Outros aspectos podem ter refletido negativamente nas taxas de reclamação, sabe-se que desde 2015, o sistema de saúde privado tem enfrentado uma redução no número de operadoras vigentes e de beneficiários em geral. A saída destes sujeitos impacta diretamente naqueles que permanecem nos planos, pois favorece o uso do método de rateio para o estabelecimento de preços, conforme previsto pela ANS. Este configura-se pela divisão do valor total das despesas entre todos os beneficiários do plano ${ }^{27}$, aumentando assim o ônus aos que permanecem com a adesão.

Cita-se também o problema com a chamada "seleção adversa", cujos valores elevados dos planos de saúde fazem com que os indivíduos que apresentam menores chances de adoecer não os contratem, permanecendo apenas o que realmente demandam por assistência à saúde, fazendo com que os valores aumentem ainda mais ${ }^{25}$. Frente a estes fatos, cria-se o cenário ideal para a insatisfação dos beneficiários, como visto neste estudo, em que o aumento da mensalidade se configurou como o terceiro motivo mais prevalente para as reclamações de idosos recebidas pela ANS. 
Frente ao exposto, pode-se observar a crescente insatisfação dos beneficiários idosos sobre a saúde suplementar, refletidas nas reclamações encaminhadas à ANS. A constatação de que o conflito de interesse entre as operadoras de planos de saúde e beneficiários afeta em especial os idosos, é de grande relevância para a definição de políticas regulatórias e de saúde para este setor ${ }^{10}$.

Qualquer política que o setor de saúde suplementar adote deve valorizar o envelhecimento saudável, a melhoria da capacidade funcional e a prevenção de doenças. Frente à possibilidade de uma crise de financiamento e reestruturação do setor, faz-se necessário abordar à saúde de forma preventiva e integral, associando o planejamento das ações em saúde ${ }^{28}$. Nessa perspectiva, torna-se um desafio para a ANS incorporar novas práticas para melhorar o atendimento ao público idoso, pprincipalmente no âmbito da promoção e prevenção de saúde, para que o desenvolvimento de doenças crônicas não potencialize as medidas adotadas para posterior regulação, evidenciando a necessidade de modificação nas práticas do cuidado com o idoso nesse setor.

Vale mencionar algumas limitações presentes neste estudo que devem ser consideradas na interpretação dos resultados. Utilizou-se apenas o banco de dados de reclamações da ANS, desconsiderando outros canais utilizados pelos beneficiários, além dos problemas envolvendo bancos de dados secundários que, por vezes, apresentam registros incompletos.

\section{Colaboradores}

NC Sousa participou do delineamento, análise, interpretação, redação e revisão crítica do conteúdo. RR Oliveira participou no delineamento, orientação do estudo, análise, interpretação e discussão dos dados e da revisão final do artigo. MA Salci orientou o estudo, participou na discussão dos dados e revisão final do artigo. L Carreira, TFCS Rodrigues e CAT Radovanovic contribuíram para a revisão crítica do conteúdo. 


\section{Referências}

1. Oliveira DF, Kornis GEM. A política de qualificação da saúde suplementar no Brasil: uma revisão crítica do índice de desempenho da saúde suplementar. Physis 2017; 27(2):207-231.

2. Brasil. Lei no 9.656, de 3 de junho de 1998. Dispõe sobre os planos e seguros privados de assistência à saúde. Diário Oficial da União 1998; 3 de junho.

3. Brasil. Lei $\mathrm{n}^{\circ}$ 9.961, de 28 de janeiro de 2000. Cria a Agência Nacional de Saúde Suplementar - ANS e dá outras providências 2000. Diário Oficial da União 2000; 28 de janeiro.

4. Avelar ABA. Evolução dos custos médico-hospitalares de planos privados de assistência à saúde no Brasil. Rev Gest Sist Saude 2018; 7(1):29-43.

5. Agência Nacional de Saúde Suplementar (ANS). Dados Gerais: Beneficiários de planos privados de saúde, por cobertura assistencial [Internet]. 2018 [acessado 2018 out 16]. Disponível em: http://www.ans.gov.br/ perfil-do-setor/dados-gerais.

6. Silva HP, Caxias MCL. Benefícios para alguns, prejuízos para muitos: Razões e implicações da adoção da dupla porta de entrada em hospitais universitários. Saude Soc 2016; 25(3):808-820.

7. Morais G, Miranda D, Cruz A, Mendes G, Andrade AL, Silva D. Population aging in Brazil: current and future social challenges and consequences. Rev Bras Geriatr Gerontol 2016; 19(3):507-519.

8. Departamento de Informáticado Sistema Único de Saúde (DATASUS). Projeção da população do Brasil por sexo e idade simples: 2000-2060 [Internet]. 2019 [acessado 2019 maio 28]. Disponível em: http:// www2.datasus.gov.br/DATASUS/index.php?are$\mathrm{a}=0203 \& \mathrm{id}=692$.

9. Veras R. A urgente e imperiosa modificação no cuidado à saúde da pessoa idosa. Rev Bras Geriatr Gerontol 2015; 18(1):5-6.

10. Vieira JWM, Martins M. The elderly and healthcare plans in Brazil analysis of the complaints received by the National Regulatory Agency for Private Health Insurance and Plans. Cien Saude Colet 2015; 20(12):3817-3826.

11. Daros RF, Gomes RS, Silva FH, Lopes TC. A satisfação do beneficiário da saúde suplementar sob a perspectiva da qualidade e integralidade. Physis 2016; 26(2):525-547.

12. Brasil. Lei $\mathrm{n}^{\circ} 10.741,1^{\circ}$ de outubro de 2003. Dispõe sobre o Estatuto do Idoso e dá outras providências. Diário Oficial da União 2003; $1^{\circ}$ out.

13. Silva KL, Rodrigues AT. Health promotion in the health insurance: relationships and tensions between private plan providers, beneficiaries and state regulatory agency. Saude Soc 2015; 24(Supl. 1):193-204.

14. Berendes S, Heywood P, Oliver S, Garner P. Quality of Private and Public Ambulatory Health Care in Low and Middle Income Countries: Systematic Review of Comparative Studies. PLoS Med 2011; 8(4):e1000433.

15. Pimenta FB, Pinho L, Silveira MF, Botelho ACC. Factors associated with chronic diseases among the elderly receiving treatment under the Family Health Strategy. Cien Saude Colet 2015; 20(8):2489-2498.

16. Miranda GMD, Mendes ACG, Silva ALA. Population aging in Brazil: current and future social challenges and consequences. Rev Bras Geriatr Gerontol 2016; 19(3):507-519.
17. Monteiro Neto A. Desigualdades regionais no Brasil: características e tendências recentes. BRU 2014; 9:6781.

18. Malta DC, Stopa SR, Pereira CA, Szwarcwald CL, Oliveira M, Reis AC. Private Health Care Coverage in the Brazilian population, according to the $2013 \mathrm{Bra}-$ zilian National Health Survey. Cien Saude Colet 2017; 22(1):179-190.

19. Garcia LP, Ocké-Reis CO, Magalhães LCG, Sant'Anna AC, Freitas LRS. Gastos com planos de saúde das famílias brasileiras: estudo descritivo com dados das Pesquisas de Orçamentos Familiares 2002-2003 e 2008-2009. Cien Saude Colet 2015; 20(5):1425-1434.

20. Bahia L, Scheffer M, Poz MD, Travassos C. Private health plans with limited coverage: the updated privatizing agenda in the context of Brazil's political and economic crisis. Cad Saude Publica 2016; 32(12):1-5.

21. Scheffer M, Bahia L. Representação política e interesses particulares na saúde: A participação de empresas de planos de saúde no financiamento de campanhas eleitorais em 2014 [Internet]. 2015 [acessado 2018 nov 30]. Disponível em: https://www.abrasco.org.br/site/wpcontent/uploads/2015/02/Planos-de-Saude-e-Eleicoes-FEV-2015-1.pdf.

22. Parahyba MI, Veras R. Socio-demographic differentials in the functional decline among the elderly in Brazil. Cien Saude Colet 2008; 13(4):1257-1264.

23. Camargos MCS, Gonzaga MR. Viver mais e melhor? Estimativas de expectativa de vida saudável para a população brasileira. Cad Saude Publica 2015; 31(7):1460-1472.

24. Confortin SC, Danielewicz AL, Antes DL, Ono LM, d'Orsi E, Barbosa AR. Associação entre doenças crônicas e força de preensão manual de idosos residentes em Florianópolis-SC, Brasil. Cien Saude Colet 2018; 23(5):1675-1685.

25. Oliveira M. Supplementary Health and aging after 19 years of regulation: where are we now? Rev Bras Geriatr Gerontol 2017; 20(5):625-634.

26. Scarpino Júnior LE, Silveira SS. Acesso à saúde privada como ferramenta de cidadania : a aplicação da vedação do comportamento. Rev Jur Cesumar 2014; 14(1):73-91.

27. Resolução Normativa DC/ANS no 356 , de 3 de outubro de 2014. Dispõe sobre a concessão de autorização de funcionamento das operadoras de planos privados de assistência à saúde. Diário Oficial da União 2014; 3 out.

28. Oliveira MR, Veras RP, Cordeiro HA, Pasinato MT. A mudança de modelo assistencial de cuidado ao idoso na saúde suplementar: Identificação de seus pontoschave e obstáculos para implementação. Physis 2016; 26(4):1383-1394.

Artigo apresentado em 26/01/2019

Aprovado em 18/11/2019

Versão final apresentada em 20/11/2019

Editores-chefes: Romeu Gomes, Antônio Augusto Moura da Silva 
